\title{
Comparison of Enteral Feeding Implementation with Standards in Educational Centers, Selected Therapeutics in Babol University of Medical Sciences
}

\author{
Research Article
}

\section{Fatemeh Rezaei $^{1^{*}}$, Mohsen Hosseinzadeh Savadi ${ }^{2}$, Maedeh Faraji Douki ${ }^{3}$, Fataneh Sabz Alipour Shiadeh ${ }^{4}$}

\author{
1. Department of Nursing, Faculty Member, 2. Department of Nursing, \\ Babol Branch, Islamic Azad University, Babol, Iran. \\ 3. Department of Nursing, Razi Hospital, Qaemshahr, Iran. \\ 4. Department of Nursing, 17 Shahrivar Hospital, Amol, Iran.
}

\begin{abstract}
Introduction: In patients who are not able to feed the mouth, but the digestive system has the ability to digest food, enteral feeding is used. This method is safe and affordable for the patient, leading to maintaining the function of the digestive system, reducing the risk of infection and sepsis. However, failure to properly implement this type of feeding causes problems such as pneumonia aspiration, infection, diarrhea, blockage of the enteral feeding, and if the above feeding method is implemented in accordance with its standard, it can play a major role in reducing the burden of complications and improving the quality of life, reducing the length of hospitalization, and reducing their costs. Method: This is a cross-sectional descriptive-analytic study. The researchers studied 37 nurses working in general and nervous wards who fed patients with gastrostomy. The checklist that was verified in terms of validity and reliability was used to collect information. For data analysis, descriptive statistics (frequency and mean) and inferential statistics (paired t-test, Spearman correlation coefficient, analysis of variance with repeated observations) were used. Results: The results showed that the mean score of actions in all stages (before, during, after implementation) was significantly lower than standard $(\mathrm{p}<0.001)$. There was also a reverse relationship between the score of actions in all three stages (before, during, after implementation) with work experience and duration of work experience in the section. There was also a significant difference between the mean score of actions in all three stages between female and male ( $p$-value -0.331), so that the average score of female was higher than that of male. Also, there was a significant difference between the mean score of the actions in all three stages and the employment status of the samples. The mean score of informal people was higher than the official ones. Conclusion: According to the findings, the mean score of the measures before, during and after the implementation of feeding through gastrostomy was significantly lower than the standard $(p<0.001)$, to enteral feeding a gynecologist at gastre and intrology, a clinical nurse and a nutritionist should educate patients and their families and personnel about the preparation and storage of food, the administration of food, the care of the entrance of the enteral and the enteral feeding, in light of the problems and complications of this type of feeding [1,2]. Based on the findings of the present study, nurses' retraining, especially men nurses, nurses with more work experience and formal nurses in the field of proper nursing clinical skills such as this type of feeding is needed.
\end{abstract}

Keywords: Enteral Feeding, Nursing Department, Gastrostomy, Feeding Support .

\section{Introduction}

Feeding is an essential component of health, and it is essential for life, natural growth and development, tissue maintenance and repair, cell metabolism, organ function, energy for movement, and maintaining body temperature. The human body needs adequate food composition for cellular function, and because of various feeding roles in metabolism and health, food planning is essential in patient care programs because the disease process, age, gender, and activity of the drug substance affect the use of food and feeding needs.

\footnotetext{
*Corresponding Author:

Fatemeh Rezaei

Faculty Member, Department of Nursing,

Babol Branch, Islamic Azad University,

Babol, IRAN
}

Email: fatemehrezaei1360@yahoo.com
Oral nutrition is the most effective way to feed the patients. But anyway, when the patient is unable to feed by her mouth, another way of feeding her is needed. The best method is the internal feeding through which the food enters the digestive system through a tube [3]. The benefits of this type of feeding are to allow the stomach to act as a natural reservoir and to regulate the amount and type of food released to the intestine [4]. And because receiving food without appetite or swallowing power helps reduce the catabolism response to damage, maintain the mucosal integrity of the intestine, reduce the placement of intestinal bacteria, and improve the wound healing [5]. This method of feeding is used in situations such as loss of consciousness, inability to swallow, trauma or esophageal or stomach cancer, oral trauma, oral surgery or anorexia, or people who require extra nutrition due to a particular condition, such as burns, infections, surgery or fractures [6]. When the enteral feeding is short-lived, less than 4 weeks, the tube 
is inserted into the stomach through the nose, but when it is needed for a long time, more than 4 weeks, the tube is inserted directly into the stomach (gastrostomy) or jejunum (jejunostomy) through the stomatium created by the surgeon in the abdominal wall. Gastrostomy is one of the most useful and most stable enteral feeding techniques available to patients who need long-term feeding support, but there are many emotional, physical, mental and cultural problems for inserting, maintaining, administering and discontinuing this type of feeding. Nurses are an important source of support and information to assist the patient in choosing this type of feeding. The patient has a complicated gastrostomy such as aspiration, tube obstruction, diarrhea, nausea, vomiting, flatulence, stomatitis [4] fever or dehydration, dry mouth, weak thoracic acid, physical complications, aspiration pneumonia, which can lead to illness and death, abdominal cramps, bleeding around the tube, red and irritated skin around the stoma, thirst, weakness, high fever, decreased urinary output due to infection and dehydration, intolerance to nutrition, pale or dark skin, cough, vomiting, wheezing, restlessness, anxiety, constipation and unwanted side effects of medications [6]. Feeding contamination in this method is a major concern because studies have shown that more than $30 \%$ of foods in this method are contaminated with a variety of microorganisms, mainly due to the preparation or implementation of feeding that has been associated with major clinical infections in the patient [7]. The contamination of this type of feeding can lead to flatulence, pneumonia, aspiration, and colonization of the germs in the patient's body [8]. In this regard, Mal Towa et al., during a research on microbial contamination in a enteral feeding on 37 patients with a enteral feeding, were provided with enteral feeding sockets and a enteral feeding plug, the results showed that $29(78 \%)$ of these patients had at least one case of germs and these contaminations were related to nurses' performance and recommended that the use of nursing precautions such as good hand washing during the implementation of this feeding method and controlling the amount of residual to reduce this complication is necessary [9]. Other issues related to this method of prescribing medications are the need for nursing interventions and skills, including preparation of medication, placement of tubes, washing the tubes and examining and recognizing potential complications. If the medications are not given correctly, adverse effects such as tube obstruction, diarrhea, aspiration pneumonia, increased costs of the health care system due to prolonged hospitalization. Tube obstruction has been reported in $67-35 \%$ of cases. In a study, nurses estimated that $50 \%$ of tube obstruction was due to inappropriate administration of medications [10]. But in spite of the complications of feeding through gastrostomy, a safe method is physiologically compatible with the body, along with the creation of favorable outcomes in the disease process and is economically economical, and therefore the preferred and most popular method of feeding [5]. The implementation of enteral feeding is a special responsibility of nurses and, if accompanied by nursing care and management, will play a key role in the success of this method of nourishment [11]. Nursing care from these patients is often based on previous training and experience, so recent research, while using these guidelines, standards and recommendations should be used to carry out this type of feeding and best care of these patients [5]. In this regard, nurses should have sufficient knowledge about indications of the use of this method of feeding, common complications and nursing interventions necessary to implement this method to prevent possible complications. The quality of the procedure is directly related to the reduction of the complications caused by this type of feeding. Standard nursing interventions can minimize the incidence of nutritional complications through gastrostomy. With regard to the above, and his own experiences with colleagues, it seems that there is a difference between the way nutrition is performed through standard gastrostomy in hospitals, so we decided to review and describe the status quo and a study titled comparison of the status of the implementation of enteral feeding (gastrostomy) with standards with goals 1- determine the mean of the score of 100 before, during, and after the enteral feeding, 2comparison of the average method for performing internal feeding at different stages (before, during, after implementation), 3-determine the relationship between the score of the method of enteral feeding implementation and some demographic characteristics (age, gender, level of education, history, experience of work in the sector, employment status) in educational centers, selected treatment centers of Babol University of medical sciences.

\section{Methods}

This is a cross-sectional descriptive-analytic study that obtained information about the method of feeding implementation through gastrostomy in patients of surgical and neurosurgical units. One-step research was conducted in spring and summer of 2010. The study population was all nurses working in surgical and neurosurgical units. The research sample was nurses who had entered the study. Samples were 37 nurses working in surgical and neurosurgical departments. The information was collected through direct observation and a checklist prepared using authoritative and authoritative sources. To determine the validity, the checklist was provided to a number of faculty members of the faculty of nursing and midwifery and their validity was evaluated by evaluating the questionnaire. To analyze the research data, descriptive statistics (frequency and mean) and inferential statistics of t-test, Spearman correlation coefficient, and analysis of variance with repeated observations were used. The questionnaire consists of two parts: the first part is related to the demographic information of the units and the second part of the questionnaire is related to the standard checklist for the implementation of enteral feeding, which consisted of 45 cases and was divided into three sections before, during, after implementation, of which 13 questions were related to the actions before implementation, 15 questions related to during implementation and 17 questions related to after implementation measures. This checklist for each item 
is based on three complete criteria ( 2 score), somewhat done (1 score), not done (0 points) scored and accordingly, the average score of 2 was calculated, with the maximum score obtained in this check list 122 and the minimum score of zero, which the scores were converted to $100-0$, and the score obtained by each unit of research was compared with a constant number of 100 , which is the ideal standard for enteral feeding practice. Also, for analysis of individual questions, a constant number of 2 (full scale score is done) was compared. Then, to determine the scores, the scores were from 0-33 (weak), 33.1-66 (average) and 66.1-100 (good). The method of work was to ensure that the program executives participated in the shifts in the morning and afternoon on the basis of the work shifts of the nurses and the presence of the disease with gastrostomy with the coordination of the responsible department in the departments and the disease was subjected to enteral feeding (gastrostomy), and the checklist was filled up based on observation.

\section{Results}

Findings of the research in relation to the mean objective score (from 100), feeding care before implementation indicated that 48.7 percent of the mean score was between 66-100 means good, 45.9 percent average score, mean $33.1-66$, and 4.5 percentage of weak score have been $0-33$, and the average score was 64.2 and the standard deviation was 17.1. In relation to the mean objective score (of 100), feeding care during implementation indicated that 16.2 percent had a good score, 64.9 percent had a average score and 18.9 percent had weak score and the average of the total score was 46.03 and the standard deviation was 17.00. In relation to the mean objective score (of 100), feeding care after implementation indicated that 67.6 percent had a good score, 29.7 percent had a average score and the average of the total score was 68.8. In relation to the mean objective score (of 100), the method of feeding in three stages (before, during and after feeding implementation), the analysis of variance with repeated observations showed that the mean score of the actions in the three stages was not the same $(p<0.001)$ And single-sample t-test showed that the mean score of actions in all stages was significantly lower than standard $(100) \quad(\mathrm{p}<0.001)$ (Table 1) and paired t-test showed that the mean score of the measures before and after the implementation was significantly higher than the measures taken during the implementation $(p<0.004)$. The comparison of the mean of the total score of the total feeding method in the before, during and after feeding stages with standard (100) showed 38.7 percent of the average of the total score of three stages was good, 54.1 percent of the average score of the total score of three stages was average and 8.1 percent of the average of the total score of three stages was weak and the average of total implementation in the three stages (before, during and after feeding implementation) was 59.88 with a standard deviation of 14.4. That is, the majority of the studied units was moderate in terms of the average total score in the three stages and was far from the standard of care provided in the implementation of feeding (Table 2). Concerning the purpose of the relationship between the method of enteral feeding through gastrostomy with some demographic characteristics of Pearson correlation test, there was no significant correlation between the score of actions in all three stages and age $(p=0.1)$, but there was a significant relationship between the score of the implementation in all three stages with work experience and duration of work experience in the department $(p=0.001)$ and $t$-test showed that there was a significant difference between the mean score of implementation in all three gender $(\mathrm{P}=0.033)$. This means that the mean score of female nurses (62) was higher than the average male nurses (51) for this type of feeding. Also, t-test showed that there was a significant difference between the mean score of implementation in all three stages and the employment status of the samples (formal or informal) $(\mathrm{p}=0.03)$, which means that the mean of informal people (64) was higher than the average score of formal people (55.4).

\section{Table 1: Average score of 100 before, during and} after feeding

\begin{tabular}{|c|c|c|c|c|c|}
\hline $\begin{array}{l}\text { Statisti- } \\
\text { cal in }\end{array}$ & $\begin{array}{l}\text { Aver- } \\
\text { age }\end{array}$ & $\begin{array}{c}\text { Stand- } \\
\text { ard } \\
\text { devia- } \\
\text { tion }\end{array}$ & $\mathbf{T}$ & $\overline{\mathrm{Df}}$ & $\mathbf{P}$ \\
\hline Before & 64.24 & $\mathbf{1 7 . 0 5}$ & -12.75 & 36 & 0.001 \\
\hline During & 46.03 & 18.22 & -18.01 & 36 & 0.001 \\
\hline After & 68.75 & 16.98 & -11.18 & 36 & 0.001 \\
\hline $\begin{array}{l}\text { Total } \\
\text { stage }\end{array}$ & $\mathbf{5 9 . 8 7}$ & 14.35 & -17.00 & 36 & 0.001 \\
\hline
\end{tabular}

Table 2: Frequency distribution and mean total score in the three stages before, during and after enteral feeding implementation

\begin{tabular}{|c|c|c|}
\hline Percent & Number & $\begin{array}{c}\text { Score actions in all } \\
\text { three stages }\end{array}$ \\
\hline $\mathbf{8 . 1}$ & 3 & $0-33$ (weak) \\
\hline 54.1 & 20 & $33.1-66$ (average) \\
\hline 37.8 & 14 & $66.1-100$ (good) \\
\hline 100 & 37 & Total \\
\hline & 59.88 & Average \\
\hline & 14.4 & Standard deviation \\
\hline
\end{tabular}

\section{Discussion}

The findings of this study showed that there are several main problems associated with feeding through gastrostomy:

- The method of conducting enteral feeding is far from its standard method, ie, the majority of the units are moderately modest in terms of how this type of feeding is performed (in all three steps before feeding, during feeding and after feeding).

- Work experience in the reverse side effect on the 
method of performing this type of feeding, in other words, will be weaker by increasing the experience of working practices.

- There is a significant difference between the method of implementation of this type of feeding and gender and the status of nurses' employment, meaning that female nurses perform better than male nurses in terms of practice, as well as formal nurses are weaker compared to informal nurses in terms of implementation of this kind of feeding.

In relation to the results, we refer to several related studies: Mateo also states in his study, "The Nursing Department of Enteral Feeding Patients" that the nursing measures performed on nourishment are very different between nurses and are different from the standard of care [12]. Robert (2003) also states that the role of nurses in the success enteral feeding support is definitive, and in this regard, the existence of executive protocols and training sessions for the implementation of the enteral feeding method is desirable [13]. Peggy Gonther (2010) also states that the safety of a disease that is feeding supplemented depends on nursing care and the correct implementation of the nurse's method and diagnosis of potential harm to the patient, because if these things are not properly executed, they can lead to serious and deadly complications [14]. In a study by Borelget et al. (2007) on the knowledge of nurses on the implementation of enteral feeding, it was noted that standard feeding is essential for enteral feeding, and the implementation of this methodology is correct due to the diversity of medical and inadequate knowledge nurses are in trouble. In addition, most studies on enteral feeding in medical and feeding journals are not studied by nurses in the sector. In this study, nourishment was performed correctly for nursing students. The results showed that their knowledge increased from 45 percent before training to 84 percent. Nurses in the study stated that the findings of this study were very surprised because they thought their knowledge was sufficient to carry out this kind of feeding. The items that were considered for the training of nurses in the field were identical in the research checklist [15]. Marshall and West (2006) also state that nurses play a major role in the proper management of enteral feeding, but nurses' skills are different and inadequate nursing knowledge has been reported in relation to the type of feeding [16]. In this regard, Caroline (2009) also states that the implementation of enteral feeding according to the protocol and standard is the best attempt that every institution can do to achieve the optimal outcome in patients, which can reduce the duration of hospitalization and reduce mortality, because feeding support is a critical component and a valuable strategy in managing patients, nurses play a major role in the management of enteral feeding. Nurses are in a good position to carry out enteral feeding for patients with the support of feeding experts and other relevant professionals. Therefore, increasing the knowledge of nurses in order to achieve optimal results in enteral feeding is necessary because knowledge of nurses has a direct impact on the success of feeding support programs, including enteral feeding [17]. Oscar (2010) and his colleagues have also considered the use of new knowledge for the proper conduct of nursing procedures and the quality of health care, and the nurses involved in her study all emphasized that their knowledge of the ability to examine and recognize the status and skills correctly [18]. In a research conducted by Hurong Leo et al. (2010) on 308 male nurses working in clinical settings, one of the effective factors in the quality of care provided by male nurses was the emotional problems of these people in relation to nursing profession due to the role of traditional masculinity in society, however, as much as giving male nurses greater power and responsibility in their field of activities, nursing care will be provided in a better way [19], and regarding the employment status, overall, it seems that having a job stability (formalism) reduces motivation and as a result has a direct impact on the correct performance of skills. However, in this regard, nursing ethics plays a very important role in caring for good [20]. On the other hand, due to the small distance between the clinical environment and training, work experience can be effective on better care, because nursing education plays a major role in the ability to perform skills, nurses who have the best training have the best nursing care [21]. Therefore, it seems that the combination of two factors of occupational safety (formal nurses) and being away from the educational environment due to increased work experience has led to a decrease in the quality of enteral feeding implementation.

\section{References}

1. Rosadahi C \& Kowalski T.Text book of Basic Nursing.6th ed: Wolters \& Kluwer / Lippincott 2008.p.347.

2. Potter \& Perry. Stockert \& Hall. basic nursing.7th ed. mosbey Elsevier .2011.p: 906. Enteral feeding. Nursing practice clinical research 2003 vol. 99. ISS.46 .P: 57

3. Taylor \& Lillis \& Lemone-Lynn. Fundamental of nursing.7th ed. Wolters \& Kluwer 2011.p: 1185

4. Williams \& Lesslie. A review of nursing care for enteral tubes in critically ill adults: part 1 , 2004 .dec, 20 (6). P: 330-343

5. Rosadahi \& Kowalski. Basics Nursing.9th ed: wolters \& kluwer / Lippincott 2008.p.347

6. Gayle Heckenberg. Enteral Feeding: Infection control. Joanna Briggs Institute Jul 19, 2008 p: 1

7. Taylor, carol. Fundamentals of nursing.6th ed: carol wolters kluwer, Lippincott 2008. p.1451

8. Lewis and Etal. Medical surgical nursing. Mosby Elsevier 2007. P. 96

9. Phillips Nicole, Nay Rhonada. Administration of medication via enteral tubes in adults: a systematic reviews. Joanna Briggs Institute, Adelaide 2007; p.324-30

10. Andrea p.Marshalla \& Sandra H.Westb. Enteral feeding in the critically ill: Are nursing practices contributing to hypo caloric feeding? .Intensive and critical care nursing. April 2006, volume 22, Issue 2; p.95-105

11. Mateo MA: Nursing management of enteral tube feeding 2009. www.ncbi.nlm.nih.gov/pubmed 
12. Roberts et al. Nutrition support in intensive care. Crit care nurse. December; 2003, 23, No. 6; p. 4957.

13. Peggi Guenter. Safe Practices for Enteral Diet in Critical Ill Patients. Critical Care Nursing Clinics of North America, June 2010, Vol. 22 Issue 2; p.197-208

14. Bourgault et al. Development of Evidence-Based Guidelines and critical care Nurse. Knowledge of Enteral feeding crit care Nurse. August 2007. Vol. 27. No 4; p. 17-29

15. Marshal A.P., West S.H. Enteral feeding in the critically ill: are nursing practices contributing to hypoalkoric feeding? Intensive and critical care nursing 2006. 22 (2). Pp. 95-705
16. Caroline Ros, Liz McNeil and Paul Bennet. Review: Nurses can improve patient nutrition in intensive care. Journal of clinical nursing .2008.18; p.2406-2415

17. Horng lou Jiumm, Hsing Yi Yu, Sheng, Hwang Chen. factors affecting the career development of male nurses: structural equation model. Journal of Advanced Nursing 2010; vol. 66th ISS. 4. P.900

18. Awatzky JO Ann and et al. teaching excellence in nursing education: A caring journal of professional nursing 2009 vol. 25, ISS. 5. P. 260.

19. Angela Chon et al. inter professional education: the interface of nursing and social work. Journal of clinical nursing 2010. Vol. 19. Iss. 1/2. p.168. 